\title{
Questions of collaboration
}

SIR - Collaboration between researchers has been increasing for many years, but whether this is the direct result of policy initiatives or reflects a process intrinsic to the global scientific community is an open question $^{1,2}$. Bibliometric evidence derived from the Science Citation Index $(S C I)$ and produced by the Science Policy Research Unit and by CHI Research Inc. suggests that the rapid growth in institutional collaboration may have started in the late 1960 s or early 1970 s and may be due to processes intrinsic to the science system.

The figure below plots two types of institutional collaboration: international and domestic. The lower two lines depict the 1973 to 1991 trends in international collaboration with UK and US partners expressed as a percentage of UK and US $S C I$ publications respectively. Because the counting techniques differ, the data from two sources were combined and an average linear regression line was calculated. The upper two lines display trends in UK industry collaborations with all domestic institutions and with UK universities only. These are expressed as a percentage of UK industry $S C I$ publications. The regression lines for both the international and domestic collaborations have been extended backwards in time to indicate where they cross the $\mathrm{x}$-axis.

In 1993 the British government released a White Paper that placed strong emphasis on formal and informal links between the public and private sectors. Irrespective of this and other government policies it is obvious from the data that domestic collaborations with UK industry are substantial and have increased throughout the 1980 s. It can also be seen that international collaboration in the UK scientific community is greater than for the United States and has been so for some time.

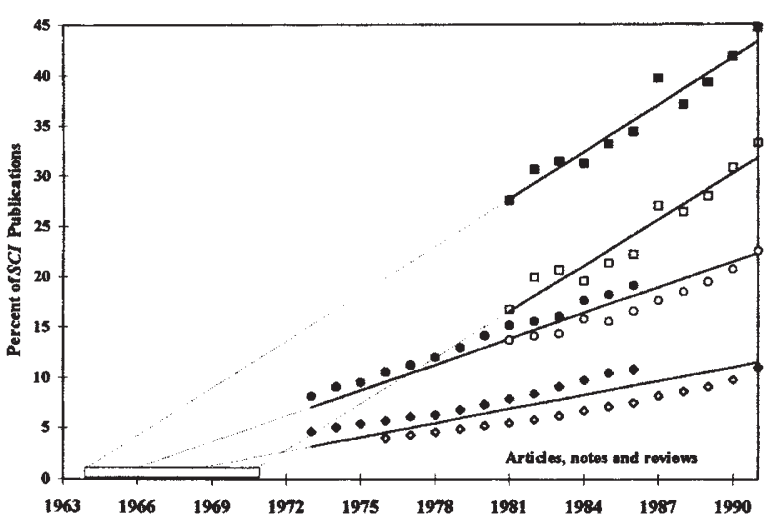

Percentage of SCl publications with institutional collaborations. UK industry domestic collaborations: $\mathbf{n}$, UK industryany domestic institution (source SPRU); $\square$, UK industry-UK university (source SPRU). UK international collaborations: (source CHI Research Inc.), O (source SPRU). USA international collaborations: $\diamond$ (source $\mathrm{CHI}$ Research Inc.); $\diamond$ (source NSF).
However, the percentage of collaboration with both countries appears to have increased at a fairly constant rate since 1973. During this time there were two Conservative and one Labour administration in the United Kingdom and four Republican and two Democratic administrations in the United States, yet there is no evidence that policy changes in either country influenced the rate of international collaboration.

For some time the effects of European Commission programmes that promote collaboration within the European Union (EU) have been of general interest to policy-makers ${ }^{3,4}$. A bibliometric analysis of UK-USA and UK-EU collaborations shows that, in 1973, about 1 per cent of UK papers involved an European partner and this proportion grew to approximately 7.5 per cent by 1991 . By comparison, in 1973 about 2 per cent of UK papers involved a US partner and this grew to 6.5 per cent by 1991 . In 1982, for the first time, a greater percentage of UK papers involved a collaboration with an EU than with a US institution. However, both growth rates were remarkably constant over this period and, on closer examination, it was found that since 1973 UK-EU collaborations had a higher growth rate than UK-US collaborations. At least at the macro level, there is no evidence for the effect of commission policy. The UK science system appears to be moving along its own trajectory.

The backward extrapolation of the collaboration trends suggest that both domestic and international collaborations may have grown at fairly constant rate for 20 to 25 years. This could be due to many factors such as the rapid expansion in international travel and telecommunications systems that began in the 1960 s, the global diffusion of scientific knowledge and personnel, the increase in the number of centres of scientific excellence, a growth in interdisciplinarity and the complex nature of research or perhaps the selforganizing nature of the science system. No matter what the causal relationship, there is an important lesson in these observations. Each country's scientific community is embedded in a global research system. At the macro level it appears to move in step with the invisible international dynamics that shape it quite independently of national policy. The global science system has a large inertia and institutional infrastruc- ture linked in a vast network - so vast in fact that it may be difficult to reshape without implementing draconian measures. In order for governments to understand their national research enterprise within the context of the global science community, they must adopt holistic and systemic investigative methods. Finally, any policy maker must conclude that scientific collaboration, including publicprivate sector collaboration, is intrinsic to the modern scientific culture. Collaboration is the rule not the exception.

\section{J.Sylvan Katz \\ Dlana Hicks}

Science Policy Research Unit,

University of Sussex,

Brighton BN1 9RF, UK

1. Katz, J. S. \& Martin, B. R. Research Policy (in the press). . Moed, H. F., de Bruin, R. E. \& Straathof, A. Report EUR 14582 (Office for Official Publications of the European Commission, Luxembourg, 1992)

3. Narin, F. \& Whitlow, E. S. Report EUR 12900 (Office for Official Publications of the European Commission. Luxembourg, 1990)

4. Moed, H. F., de Bruin, R. E., Nederhof, A. J., van Raan, A. F. J. \& Tijssen, R. J. W. Report EUR 14581 (Office for Official Publications of the European Commission, Luxembourg, 1992)

\section{Reading slides}

SIR - I am surprised that John Tiffany (Nature 374, 671; 1995) has not tried to assess the effect of the entoptic phenomenon on readability of slides. This is not a new phenomenon - it was first described by Sauvages in 1763 and confirmed by Steinbuch in 1813 and Vierordt in 1856. The effect of blue light upon the perception of this phenomenon was described separately by Rood and Reuben in 1860. I wonder with his experience in ophthalmology whether or not he has attempted mass screening of his lecture audiences for this effect upon their concentration and retentive powers?

Marcus A. Sleightholm

Seacroft Hospital,

York Road, Leeds LS14 6UH, UK

\section{Libel on Internet}

SIR - In connection with the news item "Universities in clash over Internet 'libel' allegations" (Nature 374, 297; 1995), there is as yet no innocent dissemination defence on the statute book in the United Kingdom, although the government does have legislation in draft form. There are good arguments in favour of the prevailing US approach to this issue, namely that the plaintiff should have to show that the secondary distributor (the host institution) was at fault in permitting the libel to be published. Adopting that tack would stop bulletin board defamation becoming too much of a lawyers' honeypot.

Nick Bralthwaite

Clifford Chance, 200Aldersgate Street,

London EC1A 4JJ, UK 